\title{
A Review on Increasing the Visibility of Image under Haze and Low Light Conditioning Environment
}

\author{
Priya Turkhade ${ }^{1}$, Akshay Dhande ${ }^{2}$ \\ P.G. Student, Electronics and Telecommunication Department, P. R. Patil College, Amravati, India ${ }^{1}$ \\ Associate Professor, Electronics and Telecommunication Department, P. R. Patil College, Amravati, India ${ }^{2}$
}

\begin{abstract}
This paper describes the problem of visibility of outdoor images under haze and poor light condition. Visibility is a very important issue in case of computer based surveillance, crime analysis, driver assistance system design etc. The most important challenge related to visibility is the atmospheric haze and poor lighting. The problem becomes more challenging if haze is too dense and lighting during night is extremely poor. The image processing is the vast emerging field in the era of technology of machine vision, machine intelligence and automation for real time processing or the post processing of the image captured in different atmospheric conditions. The image captured in the outdoor scene are highly degraded due to the poor lighting condition or over lighting condition or due to the presence of different suspension particle like the water droplets or dust particles. So due to these particles the irradiance coming from the object is scattered or absorbed. And hence the phenomena of haze, smoke and fog occurs. The haze removal is very essential in the field of image processing because the different computer vision algorithm assumes the input image as the original scene radiance or scene reflectance. But in most outdoor processing the images are degraded due to hazy, hence the input image is hazy image not the original radiance. In this paper we presented a technique Dark channel prior and Adaptive Histogram Equalization to improve visibility of outdoor images for different atmospheric condition.
\end{abstract}

Keywords: haze, poor lighting, dark channel prior method, adaptive histogram equalization.

\section{INTRODUCTION}

Poor visibility conditions due to bad weather, dust and smoke severely affect the performance of vision systems. In the atmospheric absorption and scattering, the emission of rays received by the camera from the scene point is attenuated along the line of sight and the incoming light is blended with the air light. This phenomenon, called haze or fog, can significantly degrade the visibility of the scene. Most computer vision applications, such as image segmentation and object tracking, usually suffer from the poor visibility of the hazy images. Therefore haze removal is highly desired in many practical applications.

Image Enhancement is the improvement of digital image quality, without knowledge about the source of degradation. The aim of image processing is to use data contained in the image to enable the system to understand, recognize and interpret the processed information available from the image pattern. Image Enhancement is the improvement of digital image quality, without knowledge about the source of degradation. Image Enhancement is the technique to improve the interpretability or perception of information in images for human viewers. In most outdoor processing the images are degraded due to hazy, hence the input image is hazy image not the original radiance. If the haze can be removed then the scene will have proper brightness, contrast and the information contents in the image will be high. The haze removal process is very complicated because the haze depends upon the unknown depth of the object in the scene. The other problem which has been considered is enhancement of image when it is captured under night condition. In this case the object is rarely visible and hence the captured image has less amount of information. To resolve these problems we proposed the method of haze removal using dark channel prior and Adaptive Histogram Equalization. In this Dark channel prior method calculates the haziness of image and adaptive histogram equalization enhances the outdoor images in extremely poor visibility condition.

\section{LITERATURE REVIEW}

In the field of haze removal different methods are present which basically built on the priority of strong assumptions. There are different papers in which many methods have been proposed by using multiple images by different authors are as follows.

Robby T. Tony [1].They have been proposed method which is based on two basic observations: first, images with enhanced visibility (or clear-day images) have more contrast than images plagued by bad weather; second, air light whose variation mainly depends on the distance of objects to the viewer, tends to be smooth. The method does not require the geometrical information of the input image, and is applicable for both colours and gray images.

Srinivasa G. Narasimhan and Shree K. Nayar [2].They have been presented a physics-based model that describes the appearances of scenes in uniform bad weather conditions. Changes in intensities of scene points under 
different weather conditions provide simple constraints to detect depth discontinuities in the scene and also to compute scene structure.

This paper [3] presented an inexpensive analytic model that approximates full spectrum daylight for various atmospheric conditions. These conditions are parameterized using terms that users can either measure or estimate. It also presents an inexpensive analytic model that approximates the effects of atmosphere (aerial perspective). These models are fielded in a number of conditions and intermediate results verified against standard literature from atmospheric science. The importance of the phenomena modelled in this paper is emphasized in the psychology and art literature.

In this paper [4] a principal component (PC)-based haze masking (PCHM) method is developed for the masking of haze in visible remote sensing images covering land surfaces at middle latitudes. Developed an haze masking method by using only the visible channel are highly correlated and haze can be easily confused with land surfaces in all these channels. It is only suitable for locally regional Haze masking where locally means that there must be clear surfaces existing in the scene and regional means that resolution is not very large.

\section{III.PROPOSED WORK}

The image captured in the outdoor scene are highly degraded due to the poor lighting condition or over lighting condition or due to the presence of different suspension particle like the water droplets or dust particles. So due to these particles the irradiance coming from the object is scattered or absorbed. And hence the phenomena of haze, smoke and fog occurs. So the images are degraded and the colours, contrast are shifted from its original irradiance at the time of capture of the image. The following fig shows the block diagram of proposed work which will used to remove the haze and to enhanced the image.

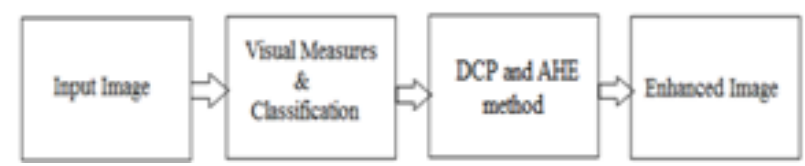

Fig 1: Block Diagram of Proposed Work.

By taking different types of image first detect the type of image and classify the image i.e. is it degraded by haze or low light. After that by it will detect the amount of haziness present in the input hazy image. On the basis of that the image is degraded by haze or low light applies the proper method one is Dark channel prior method (DCP) and other one is Adaptive histogram equalization (AHE). After applying method we get enhanced image. The basic block diagram of proposed work consists of four blocks: Input Image, Visual measures \& classification, DCP and AHE method, Enhanced image.

\section{A. Input Image}

The haze removal is very essential in the field of image processing because the different computer vision algorithm assumes the input image as the original scene radiance or scene reflectance. But in most outdoor processing the images are degraded due to hazy, hence the input image is hazy image not the original radiance. If the haze can be removed then the scene will have proper brightness, contrast and the information contents in the image will be high. The haze removal process is very complicated because the haze depends upon the unknown depth of the object in the scene.

\section{B. Visual Measures \& Classification}

In order to detect, whether the image is degraded by haze during day light or it is degraded due to insufficient lighting during night time. Based upon that it will classify the type of image. The images captured in the outdoor hazy condition suffer in the deviation of different colour channel of the RGB components present in a pixel of the image. But the sky region have the high intensity as compared to the object which we capture. So the non-sky region of the image have the lower intensity of the pixel value and hence the image may be considered as the hazy image due to the deviation of colour of the different objects present in the image. Once the type of degradation is decided, then the corresponding enhancement algorithms can be used to enhance the scene visibility.

C. Dark channel prior and Adaptive histogram equalization

when the image is a haze-free, then the minimum intensity of the different pixel value is very low in numbers. But when the image is a hazy one then the number of pixel having intensity low are high. According to that we will apply following method.

1. Dark channel prior: The concept of dark channel prior is based on our observation of outdoor haze-free images. As per the prior, in most of the local regions in haze- free images that aren't sky, very often some pixels in at least one of its colour channels(RGB) have very low intensity or close to zero. The low intensity of the dark channel is mainly due to three factors: a) shadows, e.g., the shadowing of cars, buildings and the inside of windows in cityscape images or the shadows of leaves, trees and rocks in landscape images b) colourful objects or surfaces, e.g., any object with low reflectance in any colour channel (for example, green grass, tree, plant, red or yellow flower, leaf and blue water surface) will result in low values in the dark channel; c) dark objects or surfaces ,e.g. dark tree trunks and stones. As the natural outdoor images are usually colourful and full of shadows so the dark channels of these images are really dark.

2. Adaptive Histogram Equalization: Adaptive histogram equalization (AHE) is a computer image processing technique used to improve contrast in images. It differs from ordinary histogram equalization in the respect that the adaptive method computes several histograms, each corresponding to a distinct section of the image, and uses them to redistribute the lightness values of the image. It is therefore suitable for improving the local Ordinary histogram equalization uses the same transformation derived from the image histogram to transform all pixels. This works well when the distribution of pixel values is similar throughout the image. However, when the image 
contains regions that are significantly lighter or darker than most of the image, the contrast in those regions will not be sufficiently enhanced. Adaptive histogram equalization (AHE) improves on this by transforming each pixel with a transformation function derived from a neighbourhood region.

Following are the design steps to obtain the proposed work.

Step 1: Enhancement of Hazy and Low Lighting Images

Step 2: Algorithm to Detect the Type of Images: In order to detect whether the image is degraded by haze during day light or it is degraded due to insufficient lighting during night time.

Step 3: Then it will check the image is degraded by haze else the image is degraded due to lighting of scene during night.

Step 4: Once the type of degradation is decided, then the Corresponding enhancement algorithms can be used to enhance the scene visibility.

Step 5: Apply Dark Channel Prior Method and Adaptive Histogram equalization to de-haze the image and enhanced the image

Step 6: The images captured in the outdoor hazy condition Suffer in the deviation of different colour channel of the RGB components present in pixel of the image.

Step 7: When the image is a hazy one then the number of pixel having intensity low. So according to the dark channel prior method the amount of minimum intensity value can be calculated.

Step 8: Enhancement of Night Images: Increasing Visibility in Night colour channel. The dark channel prior method can be implemented to the low-light images or night images after the inversion of the input images.

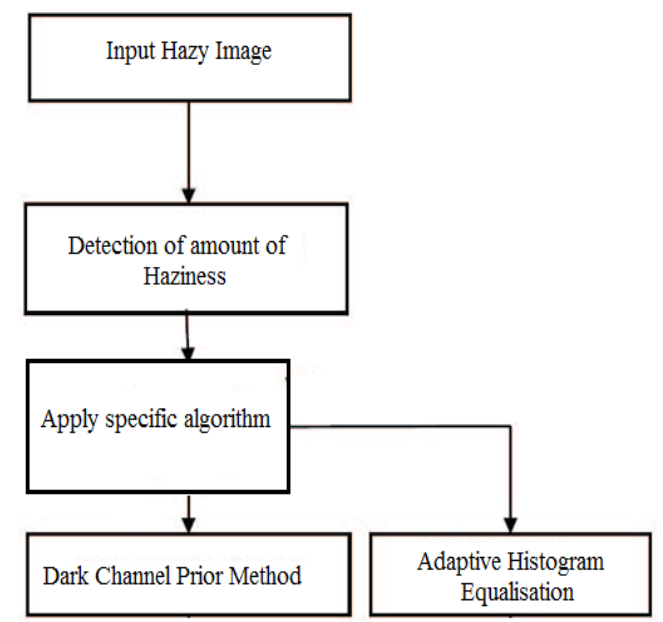

Fig 2: Flow diagram of the proposed work

\section{Enhanced Image}

Using above method we will get de-hazed or enhanced image which is degraded by haze during day light or it is degraded due to insufficient lighting during night time.

\section{IV.CONCLSION}

In this paper we proposed algorithm which will first detect and then process with proper algorithm for haze and low lighting condition. This proposed work eliminates the drawbacks arising in other existing methods. The image captured in the outdoor scene are highly degraded due to the poor lighting condition or over lighting condition or due to the presence of different suspension particle like the water droplets or dust particles. So due to these particles the irradiance coming from the object is scattered or absorbed. And hence the phenomena of haze, smoke and fog occurs. So the images are degraded and the colour, contrast are shifted from its original irradiance at the time of capture of the image. If the image is de-hazed then it can be processed and can be implemented it the field of the computer vision analysis and robotics. The proposed approach will accomplishes a higher degree of visibility recovery for images captured in extreme haze condition, as well as low light condition.

\section{REFERENCES}

[1] R. Tan. "Visibility in Bad Weather from A Single Image," in Proc. IEEE Conf. Computer Vision and Pattern Recognition., Anchorage, Alaska, pp. 1-8, Jun. 2008.

[2] G. Narasimhan and S. K. Nayar, "Contrast Restoration of Weather Degraded Images," IEEE Trans. Pattern Analysis and Machine Intelligence, vol. 25, no.6 pp. 713-724, June 2003.

[3] A.J. Preetham, P.Shirley, and B.Smits, "A Practical Analytic Model for Daylight,” Proc. ACM SIGGRAPH '99, 1999.

[4] A Principal Component Based Haze Masking Method for Visible Images Huifang Li, Liangpei Zhang,Senior Member, IEEE, and Huanfeng Shen, Senior Member, IEEE,vol.11,no.5,may2014

[5] S.G. Narasimhan and S.K. Nayar, "Chromatic Framework for Vision in Bad Weather," Proc. IEEE Conf. Computer Vision and Pattern Recognition, vol. 1, pp. 598-605, June 2000.

[6] Quality Assessment for Comparing Image Enhancement Algorithms Zhengying Chen, Tingting Jiang and Yonghong Tian* School of Electronics Engineering and Computer Science, Peking University

[7] S.G. Narasimhan and S.K. Nayar, "Vision and the Atmosphere," Int'l J. Computer Vision, vol. 48, pp. 233-254, 2002.

[8] R. Fattal."Single Image Dehazing,"in ACM SIGGRAPH '08, Los Angeles, CA Aug. 2008.

[9] K. He, J. Sun, and X. Tang. "Single Image Haze Removal Using Dark Channel Prior," in Proc. IEEE Conf. Computer Vision and Pattern Recognition. Miami, FL, pp. 1956-1963, Jun. 2009.

[10] S.G. Narasimhan and S.K. Nayar, "Interactive De weathering of an Image Using Physical Models," Proc. IEEE Workshop Colour and Photometric Methods in Computer Vision, in Conjunction with IEEE Int'l Conf. Computer Vision, Oct. 2003.

[11] X. Dong, G. Wang, Y. Pang, W. Li, J. Wen, W. Meng, and Y. Lu, "Fast efficient algorithm for enhancement of low lighting video," in Multimedia and Expo (ICME), 2011 IEEE International Conference on. IEEE, 2011.

[12] Wei-Jheng, Wang Bo-Hao, Chen Shih-Chia Huang "A Novel Visibility Restoration Algorithm for Single Hazy Images" International Conference on Systems, Man, and Cybernetics pages 847-851 IEEE 2013.

[13] Enhancement of imagery in poor visibility conditions Glenn Woodella, Daniel J. Jobsona, Zia-ur Rahmanb, Glenn Hinesa a NASA Langley Research Center, Hampton, VA College of William and Mary, Williamsburg, VA.

[14] Yishu Zhai, Dongjiang Ji “ Single image dehazing for visibility improvement "Dept. of Science, Tianjin University of Technology and Education, Tianjin.

[15] Eugene Hsu1 Tom Mertens Sylvain Paris Shai Avidan Fr'edo Durand Massachusetts "Light Mixture Estimation for Spatially Varying White Balance" Institute of Technology, Computer Science and Artificial Intelligence Laboratory Hasselt University. 